\title{
Beitrag zur Kenntnis der Nuclealreaktion in Pflanzen
}

\author{
Von \\ A. Kiesel und H. Doinikowa \\ Aus dem Laboratorium für Pflanzenbiochemie der Universität Moskau
}

Die Nuclealreaktion hat bei Zellkernforschungen ganz allgemeine Anwendung gefunden und zwar nicht allein für tierische, sondern anch für pflanzliche Objekte. Thymonucleinsäure, richtiger Desoxypentosenukleinsäure, ist dadurch in einer ganzen Reihe pflanzlicher Lebewesen verschiedener systematischer Stellung nachgewiesen worden, wobei jedoch Fälle bekannt wurden, wo die Nuclealreaktion negativ ausfiel.

Eine sehr grolse Anzahl von Phanerogamen wurde von F. BoAs und O. BIECHELE geprüft. Die meist mit Blattepidermiszellen ausgeführte Nuclealprobe ergab öfters ein negatives Resultat, woraus der Schluß gezogen wurde, daß das Vorhandensein der Desoxypentosenukleinsäure in Pflanzen nicht allgemein ist und für gewisse Pflanzen ein Artmerkmal bildet, also in systematischer Hinsicht auswertbar ist. Bei näherer Betrachtung der sich positiv oder negativ der Nuclealreaktion gegenüber verhaltenden Pflanzen ist jedoch kein sichtbares Verhältnis zum Pflanzensystem zu finden und man erhält eher den unliebsamen Eindruck, es sei der Ausfall der Reaktion einfach von irgendwelchen zufälligen Umständen abhängig. Dies kann uns jedoch keinenfalls zufrieden stellen und in der Suche nach einer annehmbarer Erklärung wäre einige Schuld an der Verwirrung dem Fehlen näherer Angaben über das Alter der geprüften Objekte oder über die Jahreszeit ihres Einsammelns zuzuschreiben.

Die Aufgabe vorliegender Arbeit war unsere Kenntnisse über die Verbreitung der Nuclealprobe in Pflanzen etwas zu erweitern, indem einerseits verschiedene Pflanzengruppen, andererseits verschiedene Organe und Objekte verschiedenen Alters untersucht wurden. In letztem Falle waren die von BoAs und BIECHELE als negativ-nucleal bezeichneten Pflanzen besonders günstige Objekte. Embryonale und aktiv wachsende Pflanzenteile stellten unter Berücksichtigung der Angaben von W. BERG der Aufmerksamkeit besonders wertes Material vor. Die von J. BRACHET in den Vordergrund gebrachte Frage über die Umbildung der Pentose- in die Desoxypentosenukleinsäure im Lauf der Entwickelung gibt uns ebenfalls Anlaß Altersunterschiede in Betracht zu ziehen. 
Wir fixierten unser Material beim Einsammeln mit Alkohol. Die Nuclealprobe wurde genau nach den Angaben von FEULGEN durchgeführt, wobei als positiv nur die im Laufe von 15, höchstens 30 Minuten eintretende Färbung bezeichnet wurde. Die hauptsächlichsten Resultate sind in den Tabellen 1 und 2 zusammengestellt.

Tabelle 1

\begin{tabular}{|c|c|c|c|}
\hline Klasse & Gattung, Art & Organ & \\
\hline $\begin{array}{l}\text { Cyanophyceae } \\
\text { Chlorophyceae } \\
\text { Conjugatae } \\
\text { Diatomeae } \\
\text { Phaeophyceae } \\
\text { Rhodophyceae } \\
\text { Filicinae } \\
\text { Equisetinae } \\
\text { Lycopodinae } \\
\text { Gymnospermae }\end{array}$ & $\begin{array}{l}\text { Oscillatorium spec. } \\
\text { Tolypothrix spec. } \\
\text { Cladophora rupestris } \\
\text { Spirogyra spec. } \\
\text { Rhabdonema spec. } \\
\text { Nitzschia spec. } \\
\text { Pylaiella litoralis } \\
\text { Polysiphonium urceolatum } \\
\text { Pteris aquilina } \\
\text { Aspidium filix mas } \\
\text { Eqü̈setum "̈iemale } \\
\text { Lycopodium complanatum } \\
\text { Ceratozamia miqueliana } \\
\text { Cedrus libanensis" } \\
\text { Pinus insiguis } \\
\text { Allium Cepa } \\
\text { Magnolia" grandiflora } \\
\text { Chrysanthemum indicum } \\
\text { Phaseolus vulgaris } \\
\text { Pisum sativum } \\
\text { Soja hispida } \\
\text { Solanum dulcamara }\end{array}$ & $\begin{array}{l}\text { Thallus } \\
\quad, \\
\text {," } \\
\text {," } \\
\text { Sporangium } \\
\text { Thallus } \\
\text { Rh"̈,om } \\
\text { Wedel } \\
\text { Unreife Sporen } \\
\text { Sporophyll } \\
\text { Unreife Sporen } \\
\text { Sporophyll } \\
\text { Unreife Spporen } \\
\text { Samenanlage } \\
\text { Macrosporangium } \\
\text { Pollen } \\
\text { Sproßscheitel } \\
\text { Zwiebelschuppen } \\
\text { Fruchtknoten } \\
\text { Sproßscheitel } \\
\text { Blatt } \\
\text { Samenkeim } \\
\text { Junges Blatt } \\
\text { Samenkeim } \\
\text { Fruchtfleisch }\end{array}$ & $\begin{array}{l}- \\
+ \\
\frac{+}{+} \\
+ \\
+ \\
+ \\
+ \\
+ \\
+ \\
+ \\
+ \\
+ \\
+ \\
+ \\
+ \\
+ \\
+ \\
+ \\
+ \\
+ \\
+ \\
+ \\
+\end{array}$ \\
\hline
\end{tabular}

Positive Nuclealreaktion wurde in allen untersuchten Pflanzengruppen aufgefunden, so daß wohl von dem Gebrauch der Nuclealreaktion für systematische Zwecke für größere Gruppen nicht die Rede sein kann. Freilich bildete unter den Algenzellkernen der ruhende Zellkern von Spirogyra eine Ausnahme. Bekanntlich fehlen diesem Zellkern auch die sonst übliche Kernfärbung mit Farbstoffen. Unser Befund bestätigt die Angaben von PETTA, der zugleich auch bei Zygnema das Fehlen der Nuclealreaktion feststellte. Die vorhandenen Angaben von Poljansky und Petrouchewsky über die positive Nuclealreaktion bei kernlosen Cyanophyceen konnten wir dagegen nicht bestätigen. Hierbei muß jedoch berücksichtigt werden, daß Alter und Entwickelungsstadium der Zellen in unserem Falle nicht dieselben sein konnten, wie bei den genannten Forschern. Unreife Sporen zeigten sämmtlich negative Nuclealprobe an. Dennoch kann bis auf weiteres nicht behauptet werden, daß dies vom Reifungsstadium abhing. 
Tabelle 2

\begin{tabular}{|c|c|c|c|c|}
\hline & \multicolumn{2}{|c|}{$\begin{array}{l}\text { Angaben von BOAS } \\
\text { und BIECHELE }\end{array}$} & \multicolumn{2}{|c|}{ Unsere Beobachtung } \\
\hline & Organ & $\begin{array}{l}\text { Nucleal- } \\
\text { reaktion }\end{array}$ & Organ & $\begin{array}{l}\text { Nucleal- } \\
\text { reaktion }\end{array}$ \\
\hline Pinus silvestris & Nadelblatt & - & Sproßscheitel & + \\
\hline ", montana & " & - & $"$ & $t$ \\
\hline $\begin{array}{l}\text { Prea excelsa } \\
\text { Magnolia pumila }\end{array}$ & Blatë & - & $"$ & $\begin{array}{l}+ \\
+\end{array}$ \\
\hline Vicia faba & Kotyledonen & - & Samenkeim & + \\
\hline Lüpinus perennis & $\begin{array}{l}\text { Wurzel } \\
\text { Kotyledonen }\end{array}$ & - & $\begin{array}{l}\text { Blatt, junge Pflanze } \\
\text { Samenkeim }\end{array}$ & $\begin{array}{l}t \\
+\end{array}$ \\
\hline ", $\quad$, & Wurzel & - & $\begin{array}{l}\text { Keimling, Wurzel } \\
\text { Keimling, Blatt }\end{array}$ & + \\
\hline ", $\quad "$, & & & Erwachsene Pflanze, Blatt &.-+ \\
\hline Onöbrychis sätiva & Blatt & - & Blatt & - \\
\hline Primula obconica & Blatt & - & Staubblatt & $\begin{array}{l}+ \\
+\end{array}$ \\
\hline$"$, & & & $\begin{array}{l}\text { Pollenkorn } \\
\text { Erwachsene Pflanze, Blatt }\end{array}$ & + \\
\hline Betula alba" & Blatt & - & Laubknospe & - \\
\hline$", \quad "$ & & & $\begin{array}{l}\text { Blatt, jung und alt. } \\
\text { Sproß }\end{array}$ & - \\
\hline , & & & Samen & + \\
\hline
\end{tabular}

In der Tab. 2 sind einige der von BoAs und BIECHELE als nuclealnegativ gefundene Pflanzen angeführt, bei denen wir dennoch positive Nuclealreaktion erhalten konnten. Außer Blattepidermiszellen wurden von uns auch andere Zellenarten untersucht, wobei Altersunterschiede von Bedeutung waren. Aktiv wachsende und entwickelungsfähige Pflanzenteile zeichneten sich stets durch stärkere positive Nuclealprobe aus, mußten deshalb größere Mengen der uns interessierenden Nucleinsäure im Zellkern enthalten.

Zusammenfassend darf wohl behauptet werden, daß es kaum Pflanzen gibt, denen die Fähigkeit der Desoxypentosenukleinsäurebildung abkommt. Bei allgemeiner Bildung kann es jedoch bestimmte Entwickelungsmomente geben, wo dem Zellkern die genannte Nucleinsäure fehlt. Dadurch wird ihre Bedeutung für das Zellenleben keinesfalls in Zweifel gezogen, es könnte jedoch vermutet werden, daß beim zeitweiligen Fehlen der Desoxypentosenukleinsäure im Zellkern ähnliche Umbildungsvorgänge verlaufen, wie sie von einer Reihe von Forschern für die ihr Reifungsstadium durchlaufenden und befruchteten Eizellen einzelner Tierarten beobachtet und von J. BRACHET analytisch festgestellt sind. Freilich wäre dabei außer der nachgewiesenen Umbildung der Pentose- in die Desoxypentosegruppe auch eine Umbildung der basischen Gruppen aufzufinden. Dies ist mit bedeutend größerer Mühe verbunden, jedoch keinesfalls aussichtslos und verspricht wichtige Aufklärungen für den Zellkernstoffwechsel zu liefern.

Zum Schluß müssen wir auf die Angaben von T. CAsperssoN hinweisen, der den negativen Ausfall der Nuclealreaktion nicht 
als einen Beweis des Fehlens von Thymonukleinsäure ansehen will, sondern ihn den störenden Einwirkungen größerer Mengen von Eiweiß zuschreibt. Bei dieser Sachlage könnten nur positiv verlaufende Färbungen maßgebend sein, negative jedoch vollständig wertlos werden. $\mathrm{Ob}$ dem auch wirklich so ist, wäre unbedingt nachzuprüfen, wozu die Absorptionsmessung im U.-V. Spektralgebiet nach T. CASPERSSON behilflich sein könnte, die freilich keine Unterscheidung zwischen verschiedenen Nukleinsäuren gestattet.

\section{Zitierte Literatur}

Berg, W. Zeitschr. f. mikr.-anat. Forschung, Bd. 7, S. 421, (1924).

Brachet, J. Archives de Biologie, T. 44. p. 519 (1933).

Brachet, J. Bullet. d. l. Soc. de chim. biolog., T. 18, p. 305, (1935).

Boas, F. und Biechele, O. Biochem. Zeit. Bd. 254, S. 467, (1932).

Caspersson, T. Biochem. Zeit., Bd. 253, S. 97, (1932).

Caspersson, T. Skandinav. Archiv. f. Physiol. Suppl. N. 8 zum 7 Bd. (1936).

Feulgen, R. und Rossenbeck, H. Zeit. f. Physiol. Chem. Bd. 135, S. 203, (1924).

Petta, H. Compt. rend. de l'acad. d. sc. Paris. T. 197, p. 88, (1933).

Poljansky, G. und Petrouchewsky. Arch. d. Protistenkunde Bd. 67, H. 1, (1929). 